\title{
Hypoglycaemia induces recruitment of non-classical monocytes and cytotoxic lymphocyte subsets in type 1 diabetes
}

\author{
Jacqueline M. Ratter ${ }^{1,2}$ (D) Hanne M. M. Rooijackers ${ }^{1} \cdot$ Cor W. M. Jacobs $^{1} \cdot$ Bastiaan E. de Galan $^{1} \cdot$ Cees J. Tack $^{1} \cdot$ \\ Rinke Stienstra ${ }^{1,2}$
}

Received: 3 April 2018 / Accepted: 1 June 2018 / Published online: 7 July 2018

(C) Springer-Verlag GmbH Germany, part of Springer Nature 2018

Keywords Hypoglycaemia · Inflammation · Type 1 diabetes

\section{Abbreviation \\ PBMC Peripheral blood mononuclear cell}

To the Editor: We recently reported enhanced ex vivo cytokine production of immune cells during hypoglycaemia in humans [1]. Chronic inflammation contributes to cardiovascular disease and atherosclerosis and may link hypoglycaemia to increased cardiovascular risk and mortality observed in individuals with diabetes [2].

Hypoglycaemia induces leucocytosis, possibly mediated by marked increases in plasma adrenaline (epinephrine) and cortisol levels. Adrenaline recruits immune cells from the marginal pool (vascular endothelium), likely by interfering with CD11a- and CX3CR1-dependent adhesion of leucocytes to the vessel wall [3]. Indeed, gene expression levels of CD11a and CX3CR1 were increased in peripheral blood mononuclear cells (PBMCs) isolated during hypoglycaemia [1].

It remains unknown which specific immune cell subsets are recruited in response to hypoglycaemia. To identify these subsets, we analysed blood samples collected during clamped euglycaemia or hypoglycaemia from seven people with type 1 diabetes (three men, age $27.1 \pm 9.7$ years, $\mathrm{HbA}_{1 \mathrm{c}} 55.9 \pm$ $8.9 \mathrm{mmol} / \mathrm{mol}[7.2 \pm 0.8 \%]$ and diabetes duration $11.4 \pm$ 5.4 years; mean \pm SD) by flow cytometry. This was a substudy of a larger study (ClinicalTrials.gov registration no.

Jacqueline M. Ratter

Jacqueline.Ratter@ radboudumc.nl

1 Department of Internal Medicine, Radboud University Medical Center, PO Box 9101, 6500 HB Nijmegen, the Netherlands

2 Division of Human Nutrition and Health, Wageningen University, Wageningen, the Netherlands
NCT03286816), for which we analysed data obtained within the control arm (for study design and endpoints, see [4]). The study was approved by the local institutional review board of Radboud University Medical Center and all study participants gave written informed consent before participation. All participants had normal awareness of hypoglycaemia (assessed by the Dutch version of the Cox questionnaire and normal counter-regulatory responses to the hypoglycaemic clamp).

Participants underwent a two-step euglycaemic ( $5.0 \mathrm{mmol} / \mathrm{l})$ hypoglycaemic $(2.8 \mathrm{mmol} / \mathrm{l})$ glucose clamp. At the end of each glycaemic phase, blood was drawn for flow cytometry and to determine catecholamine levels. Undiluted blood (50 $\mu$, BD Vacutainer K2E [EDTA], BD, Plymouth, UK) was incubated for $15 \mathrm{~min}$ at room temperature in the dark with the following antibodies (all mouse): CD16-FITC (dilution 1:5), CD56-PE (1:10) (BD Biosciences, Vianen, the Netherlands); CD14-ECD (1:10), CD3-ECD (1:10), CD3-PE (1:5), CD45-PECy7 (1:50), CD8-ECD (1:10), HLA-DRPECy5 (1:10) (Beckman Coulter, Woerden, the Netherlands); CD4-PECy7 (1:20) (ITK Diagnostics BV, Uithoorn, the Netherlands). After the addition of $1 \mathrm{ml}$ lysis buffer (BD Pharm Lyse, BD Biosciences), samples were mixed, incubated for another $10 \mathrm{~min}$ and finally measured on a Beckman Coulter FC500 flow cytometer. Single staining and fluorescence-minus-one control stains were used to determine the position of analysis gates. Flow cytometry data were analysed using Kaluza software (Beckman Coulter). Immune cell subset numbers were calculated based on percentages measured with flow cytometry and cell numbers from blood differentials measured on a Sysmex XN-450 (Sysmex, EttenLeur, the Netherlands). A Spearman's rank sum test was used for correlation analysis. Wilcoxon signed rank test was used to test differences between two related samples. All data are expressed as mean $\pm \mathrm{SD}$. A $p$ value of $<0.05$ was considered 
statistically significant. Statistical analyses were performed with SPSS version 22 (IBM, Armonk, NY, USA).

Glucose levels during the euglycaemic-hypoglycaemic clamp were maintained at $5.2 \pm 0.2$ and $2.8 \pm 0.1 \mathrm{mmol} / \mathrm{l}$, respectively. Hypoglycaemia significantly increased adrenaline levels from $0.30 \pm 0.19$ to $3.40 \pm 2.27 \mathrm{nmol} / 1(p=$ 0.018). As expected, hypoglycaemia increased leucocyte counts (Fig. 1a), which was predominantly due to increased numbers and relative percentages of lymphocytes and monocytes, but not neutrophils (Fig. 1b-g).

Elevated lymphocyte counts were mainly due to increased numbers of $\mathrm{CD}^{+} \mathrm{T}$ cells (Fig. 1i), which was reflected in decreased percentages of $\mathrm{CD}^{+}$and increased percentages of $\mathrm{CD}^{+} \mathrm{T}$ cells (Fig. 1j, k). Increased percentages of $\mathrm{CD} 8^{+} \mathrm{T}$ cells during hypoglycaemia were correlated with the increase in adrenaline levels $(r=0.786, p=0.036)$. Additionally, hypoglycaemia increased numbers and percentages of NKT cells and $\mathrm{CD} 16^{+} \mathrm{CD} 56^{\mathrm{dim}}$ cytotoxic NK cells (Fig. 11-o). Numbers of circulating CD $16^{\text {low }} \mathrm{CD} 56^{\text {bright }}$ NK cells were generally very low, and not different between the glycaemic conditions (data not shown). Hypoglycaemia increased classical $\left(\mathrm{CD} 14^{++} \mathrm{CD} 16^{-}\right)$and non-classical $\left(\mathrm{CD} 14^{+} \mathrm{CD} 16^{++}\right)$ monocyte numbers (Fig. 1p, q). However, percentages of non-classical monocytes increased, while percentages of classical monocytes decreased (Fig. 1s, t). The ratio of classical monocytes measured under hypoglycaemic vs euglycaemic conditions was negatively correlated with the increase in adrenaline levels $(r=-0.857, p=0.014)$.

Increased percentages of $\mathrm{CD}^{+} \mathrm{T}$ cells in response to hypoglycaemia are in line with previous studies demonstrating increased gene expression of $C D 8 A$ in PBMCs during hypoglycaemia [1] and are likely to be caused by increased
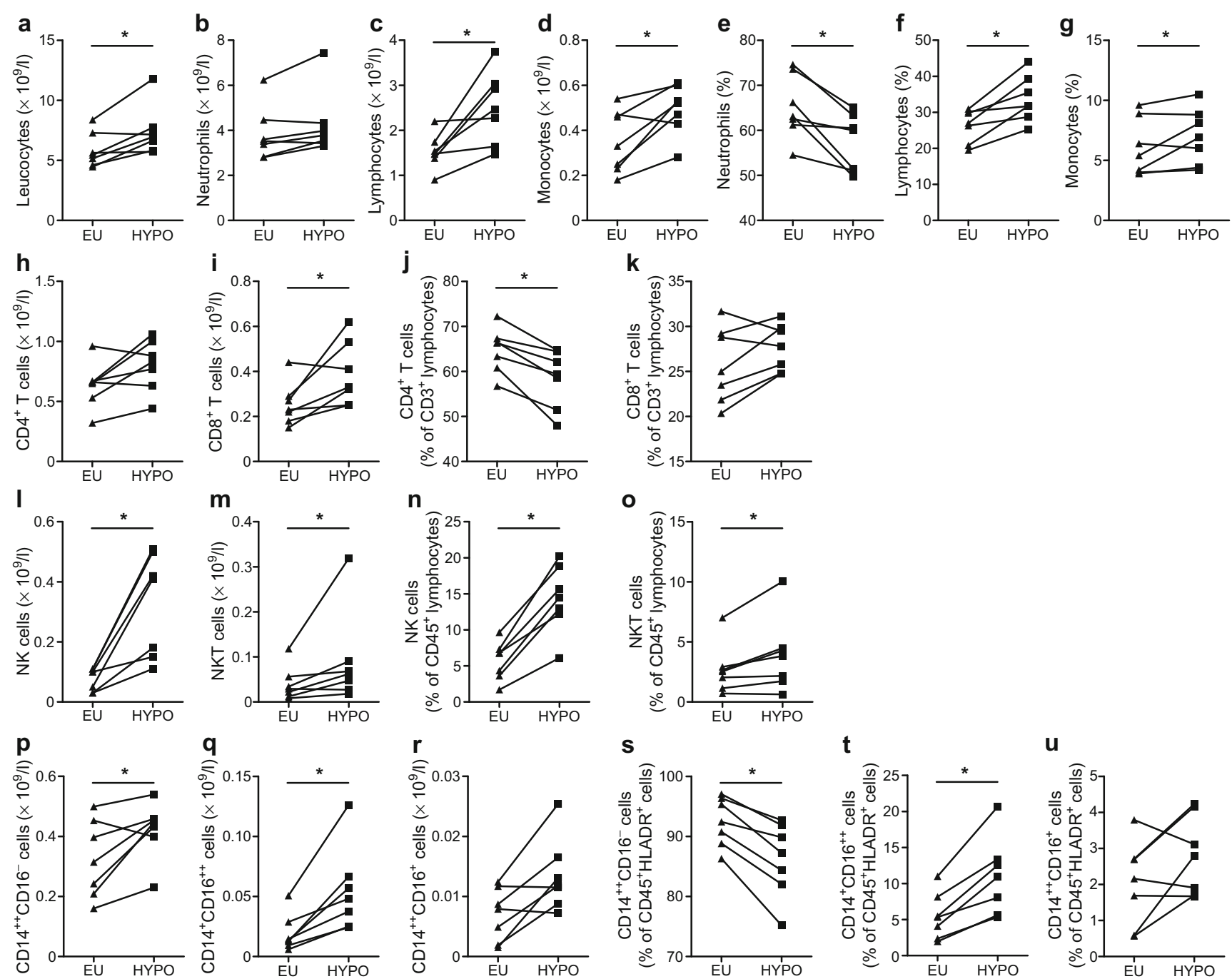

j
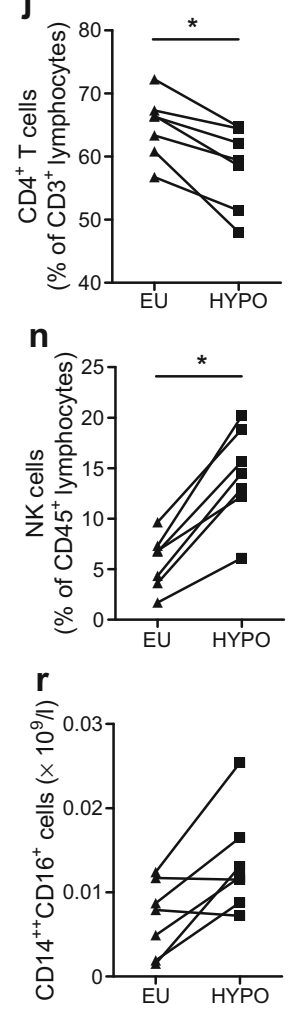

Fig. 1 Numbers of leucocytes (a), neutrophils (b), lymphocytes (c) and monocytes (d) and percentages of neutrophils (e), lymphocytes (f) and monocytes (g) measured during euglycaemia (EU) and hypoglycaemia (HYPO) in individuals with type 1 diabetes. Numbers and percentages of $\mathrm{CD}^{+} \mathrm{T}$ cells and $\mathrm{CD}^{+}$T cells $(\mathbf{h}-\mathbf{k}), \mathrm{CD} 16^{+} \mathrm{CD} 56^{\mathrm{dim}} \mathrm{NK}$ cells and NKT
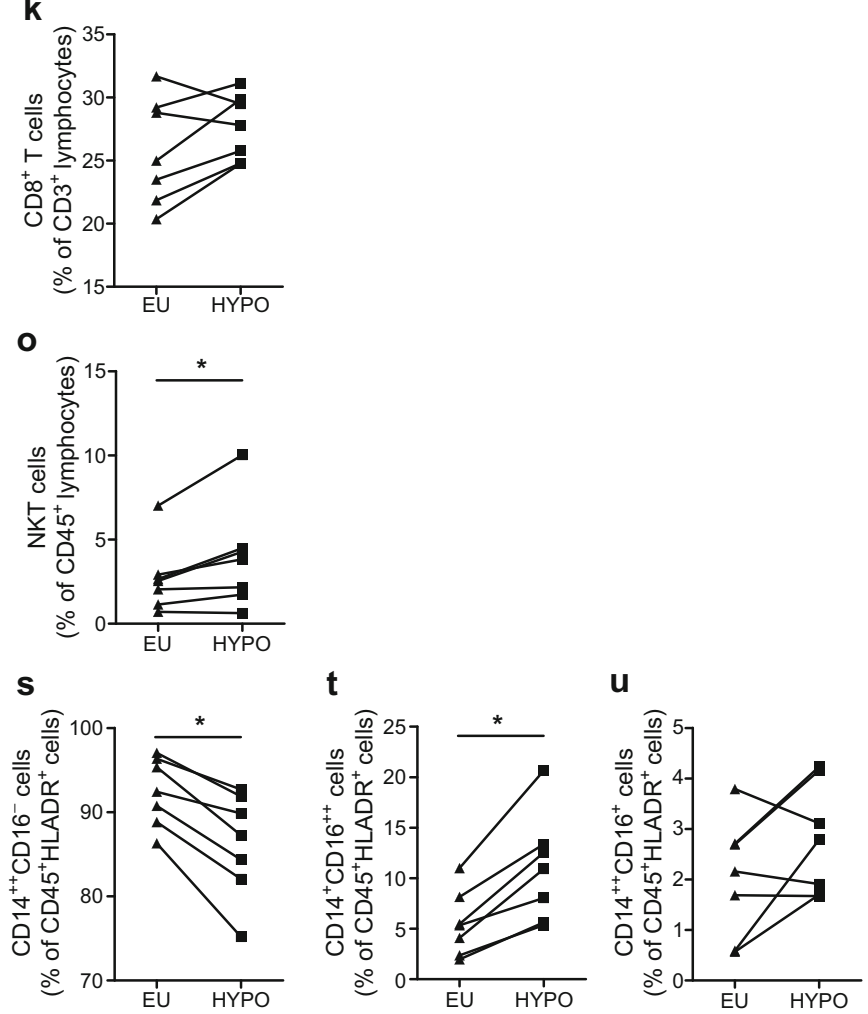

cells $(\mathbf{l}-\mathbf{o})$ and classical $\mathrm{CD} 14^{++} \mathrm{CD} 16^{-}$, non-classical CD $14^{+} \mathrm{CD} 16^{++}$and intermediate $\mathrm{CD} 14^{++} \mathrm{CD} 16^{+}$monocytes (p-u), measured during EU and HYPO in individuals with type 1 diabetes. Leucocytes were identified as $\mathrm{CD}_{4} 5^{+}$cells, T cells as $\mathrm{CD}^{+}$cells and monocytes as $\mathrm{CD} 45^{+} \mathrm{HLADR}^{+}$ cells. $* p<0.05$, Wilcoxon signed rank test 
adrenaline levels [3]. Although $\mathrm{CD} 8^{+} \mathrm{T}$ cells are atheroprotective in some situations, several pro-atherogenic effects have been demonstrated [5]. Increases in NK and NKT cells might also contribute to cardiovascular disease. NK cells were found in atherosclerotic lesions, where they increase lesion size by release of perforin and granzyme B [6]. Similarly, increased numbers of NKT cells, which can recognise lipid antigens presented via CD1d, have been observed in atherosclerotic lesions [7].

Monocyte-derived macrophages, the most abundant cells in atherosclerotic plaques, differentiate into foam cells and contribute to plaque instability. Different monocyte subsets - classical, non-classical and intermediate - have distinct inflammatory and functional properties. Although all subsets have been related to cardiovascular disease risk, there is more evidence supporting the importance of non-classical and intermediate monocytes in the development of cardiovascular disease [8]. Hypoglycaemia-induced monocytosis was mainly driven by an increase in non-classical monocytes. This may explain increased cytokine production of monocytes isolated during hypoglycaemia vs euglycaemia [1], since non-classical monocytes produce more proinflammatory cytokines [9].

The clinical implications of our findings depend on various factors and require further investigation. First, recruitment of immune cells may differ between individuals with type 1 vs type 2 diabetes and between moderate vs severe hypoglycaemia. Additionally, the persistence of the effect on changes in circulating immune cell populations is currently unknown. The timeframe over which the distribution and function of the immune cell populations return, and whether this depends on the recurrence of hypoglycaemia, remain to be determined. Frequent hypoglycaemic events may indeed induce chronic inflammation, but the reduced catecholaminergic responses following recurrent hypoglycaemia may also have a dampening effect on inflammation. Interestingly, recent studies have reported that although neuroendocrine responses were reduced by antecedent hypoglycaemia, proatherotrombotic factors were increased [10]. One may speculate that acute effects of hypoglycaemia on immune cells promote a proatherogenic state dependent on other proatherogenic factors, for example, prior exposure to (chronic) hyperglycaemia.

In conclusion, hypoglycaemia increased quantities of $\mathrm{CD}^{+} \mathrm{T}$ cells, cytotoxic NK and NKT cells and non-classical monocytes in people with type 1 diabetes. Our study further supports the concept that hypoglycaemia promotes acute inflammation in individuals with type 1 diabetes, which may contribute to a more chronic (sustained) proinflammatory state.

Acknowledgements The authors thank all volunteers for participating in this work. We are indebted to K. Saini and A. Hofboer-Kapteijns (Clinical
Research Center Nijmegen, Radboud University Medical Center, the Netherlands) for assistance during the glucose clamps.

Data availability The data generated during and/or analysed during the current study are available from the corresponding author on reasonable request.

Funding Research support from the Dutch Diabetes Research Foundation (DFN 2012.00.1542), Sanofi (unrestricted research grant) and the European Foundation for the Study of Diabetes is gratefully acknowledged. RS is supported by a VIDI-grant from The Netherlands Organisation for Scientific Research (NWO) and a senior fellowship from the Dutch Diabetes Foundation.

Duality of interest The authors declare no potential conflicts of interests relevant to this study.

Contribution statement JR, HR, BdG, CT and RS designed the study. JR performed and analysed flow cytometry experiments. HR recruited patients and performed clamps. CJ assisted in performance and analysis of flow cytometry measurements. JR and RS wrote the paper. HR, CJ, BdG and CT critically revised the paper for important intellectual content. All authors approved the final manuscript to be published. RS is the guarantor of this work.

\section{References}

1. Ratter JM, Rooijackers HM, Tack CJ et al (2017) Proinflammatory effects of hypoglycemia in humans with or without diabetes. Diabetes 66:1052-1061

2. Zoungas S, Patel A, Chalmers J et al (2010) Severe hypoglycemia and risks of vascular events and death. N Engl J Med 363: 1410-1418

3. Dimitrov S, Lange T, Born J (2010) Selective mobilization of cytotoxic leukocytes by epinephrine. J Immunol 184:503-511

4. Wiegers EC, Rooijackers HM, Tack CJ et al (2018) Effect of lactate administration on brain lactate levels during hypoglycemia in patients with type 1 diabetes. J Cereb Blood Flow Metab. https://doi. org $/ 10.1177 / 0271678 X 18775884$

5. Cochain C, Zernecke A (2016) Protective and pathogenic roles of $\mathrm{CD}^{+} \mathrm{T}$ cells in atherosclerosis. Basic Res Cardiol 111:71

6. Selathurai A, Deswaerte V, Kanellakis P et al (2014) Natural killer (NK) cells augment atherosclerosis by cytotoxic-dependent mechanisms. Cardiovasc Res 102:128-137

7. van Puijvelde GHM, Kuiper J (2017) NKT cells in cardiovascular diseases. Eur J Pharmacol 816:47-57

8. Weber C, Shantsila E, Hristov M et al (2016) Role and analysis of monocyte subsets in cardiovascular disease. Joint consensus document of the European Society of Cardiology (ESC) Working Groups "Atherosclerosis \& Vascular Biology" and "Thrombosis". Thromb Haemost 116:626-637

9. Mukherjee R, Kanti Barman P, Kumar Thatoi P, Tripathy R, Kumar Das B, Ravindran B (2015) Non-classical monocytes display inflammatory features: validation in sepsis and systemic lupus erythematous. Sci Rep 5:13886

10. Joy NG, Tate DB, Younk LM, Davis SN (2015) Effects of acute and antecedent hypoglycemia on endothelial function and markers of atherothrombotic balance in healthy humans. Diabetes 64:2571-2580 